\title{
Calculation of Unbalanced Tension of Transmission Line by Image Recognition Method of Insulator Deflection Angle
}

\author{
Yehe Shi \\ School of Mechanical Engineering, North China Electric Power University, Baoding 071003, \\ China \\ syhkxmyt@126.com
}

\begin{abstract}
Keywords: Insulator Deflection Angle; Image Processing Technology; Calculation of Unbalanced Tension
\end{abstract}

\begin{abstract}
With the development of the transmission line, it is more and more important to analyze the unbalanced tension of the tower. The non-contact method of measuring the insulator deflection angle uses image processing techniques, and the experimental results show that this method has better recognition speed and accuracy. First, we capture insulator image by using a camera fixed on the tower; then use image filtering, image thinning techniques for image pre-processing. Finally, we extract the central axis of insulator using Hough transformation, get the insulator deflection angle and analyze the unbalanced tension. It can be worked out by the corresponding computer program.
\end{abstract}

\section{Introduction}

Transmission lines are a very important part of the electric power system. Uneven tension is one of the most important factors that can endanger the safe and stability of transmission lines [1].Through the basic equation; we can obtain the relationship between the wire tension and the insulator deflection angle. So the uneven tension of transmission lines can be monitored by measuring insulator deflection angle. In recent years, digital video monitoring has been widely used in electric power system, along with image processing technology [2, 3]. The tilt angle of the insulator can be obtained by using real-time image analysis technology, then we can obtain the unbalanced tension.

The reliability of the power grid transmission largely depends on the stability of the transmission line working state. Overhead transmission lines are faced with the wind, rain, ice, lightning and other natural threat. Accidents will occur especially in bad weather condition. Calculation of unbalance tension by using image processing technology for real-time monitoring of insulator Angle can provide reference basis for transmission line accident [4].

\section{Basic Concept Statement}

\section{Unbalanced Tension Calculation.}

Unbalanced tension means the tension gap on the tower which caused by the change of weather condition. Unbalanced tension of overhead lines can cause the offset of the insulator string, so calculation of the uneven tension can be measured deviation angle of insulator string. According to the balance of force and moment equilibrium conditions, we can get the following formula:

$$
s_{i+1}=\left(\frac{G_{i}}{2 A}+\frac{l_{i} r_{i}}{2 \cos b_{i}}+\frac{l_{i+1} r_{i+1}}{2 \cos b_{i+1}}+\frac{s_{i} h_{i}}{l_{i}}+\frac{s_{i}}{d_{i}} \sqrt{l_{i}^{2}-d_{i}^{2}}\right) /\left(\frac{\sqrt{l_{i}^{2}-d_{i}^{2}}}{d_{i}}+\frac{h_{i+1}}{l_{i+1}}\right)
$$

$\mathrm{G}_{\mathrm{i}}$ : the weight of insulator strings of the $\mathrm{i}$-th span;

$\lambda_{\mathrm{i}}$ : the length of insulator strings of the i-th span;

$\delta_{\mathrm{i}}$ : the offset of insulator string of the i-th span;

A: Cross-sectional area of the conductor 


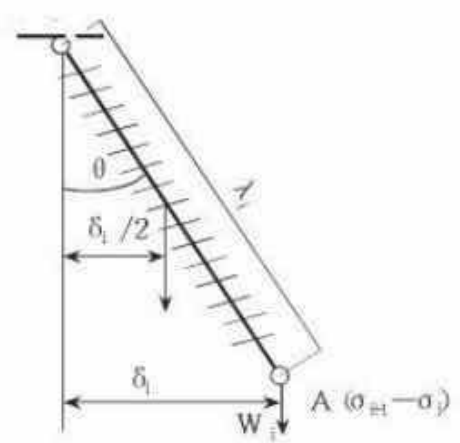

\section{Hough transforms.}

Fig.1 the offset of pendant insulator stained

Hough transform has good fault tolerance and robustness, is a kind of parameter estimation technology anchored on voting principle. Hough transform is based on the duality theorem, and it turns into the straight line detection problems in image space into the spot detection problems in parameter space. The most widely used method of linear Hough transform is to establish dual transformation between image space and parameter space based on polar coordinates.

Hough transform divides the polar coordinates in parameter space into many small lattices, namely the small case accumulators. For each point, we calculate the corresponding .The result value (rounding) fall in a small lattice, and then the value of the small lattice will add 1.Eventually we need to test the value of all of the accumulators. The small lattice of the peak corresponds to the collinear points in the parameter space The small lattice with small value generally reflect the non-collinear points, so they will be discarded.

\section{Morphological image processing}

Each pixel value of binary image can only be 0 or 1 , in practice binary image pixels can be expressed by logical value. Commonly, 0 means black and 1 means white. The target to be operated is image sets. The basic operation of mathematical morphology is that structural elements in the binary image replace the images while moving the probe.

$A$ is the image to be processed, and B is the structural element. In the process of translation of structural elements. In dilation diagram, if the structural elements and the pixel whose value is 1 overlap, then output 1 , otherwise output 0 . n erosion diagram, if they perfectly match, then output 1 , otherwise output 0 .

The morphological operations including inflation, corrosion, opening operation and closing operation. The morphological opening operation is the image corrosion operations at first then expansion operations. Opening operation can filter out spots smaller than structural elements, smooth object contour, disconnect the narrow links, and remove tiny highlights.

The morphological the closing operation is image expansion operations at first then corrosion operations. Closing operation can connect narrow gap form elongated curved mouth, and then fill the holes which are smaller than structural elements.

\section{Process of calculation of uneven tension}

The process of insulator angle measurement mainly includes the image information acquisition and preprocessing, insulator axis extraction, calculation of slope angle and unbalanced tension.

\section{Image information collection and pre-treatment}

First, to capture real-time insulator image, we fix a camera at the specified area on the tower; adjust video acquisition area. Video image preprocessing and insulator axis extraction is used to calculate the slope angle of insulator.

The purpose of image pre-processing to is removing noise and irrelevant element. It transfers the original image into suitable feature extraction in the form which the computer can distinguish. Refined image morphology transformation is used to get the insulator of bone structure. 


\section{Extraction of insulator axis and calculation of tilt Angle}

After binarization of the insulator image thinning first, we use the hough transform to detect insulator [5] the central axis, In the established coordinate system, the slope k insulator axis is the tilt angle of insulator.

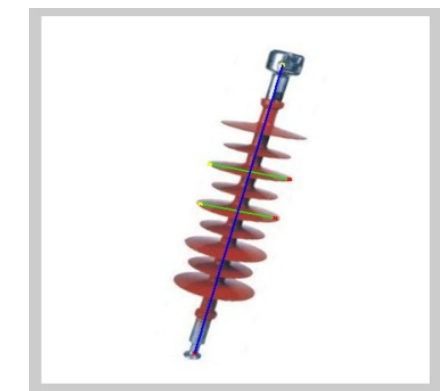

\section{Calculation of Unbalanced Tension}

Fig.2 Central axis of insulator

Based on the relationship of insulator slope angle and the uneven tension, we calculate the unbalance tension by the corresponding computer program.

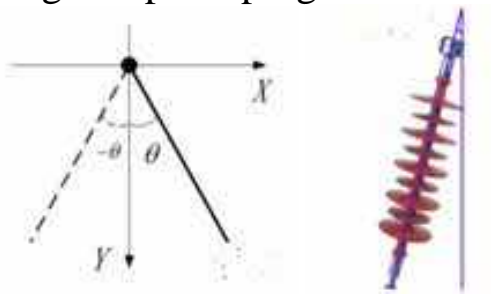

Fig.3 Insulator deflection angle

\section{Experimental Results}

Based on the above process, we work out the corresponding computer program. The system mainly consists of digital image acquisition, image processing and real-time monitoring.

Firstly we acquire insulator image, then use the technology of image pre-processing including filtering, binarization, image thinning. Hought transform is utilized to get the extract refined image of the straight line. We obtain the insulator angle in accordance with the set coordinate system. According to the actual needs, we can set the resolution of the collected images and different environmental backgrounds. Thus unbalanced Tension is obtained.

For high speed continuous detection, we need to get the video of insulator movement process first, and then deal with the video.We calculate the insulator angle of each frame, and then draw out the curve of the angle changing with time. According to insulator tilt movement of the process, real-time unbalanced tension can be obtained.

\section{Summary}

The method presented in this paper has high accuracy and practicability for the non-contact measurement of inclination of the insulator angle. We can improve the image processing methods, to speed up the extraction process and to realize real-time monitoring.

\section{References}

[1] Qingfeng Liu, Analysis and calculation of unbalanced tension of transmission line, Electrical automation equipment, 2006,01,: 93-95

[2] Weiguo Quan, Jinsha Yuan, Baoshu Li. Review on Application of image processing technology in the transmission line inspection of helicopter inspection [J]. The grid technology, 2010, 12: 204-208. 
[3] Fengjie Sun, Weixin Cui, Jinbao Zhang, Xudong Zhang, Xuedong Xiao. Application of digital video monitoring and image recognition technology in electric power system [J]. The grid technology, 2005, 05: 81-84.

[4] HaokunGuo, Sikun Heng, Zhanfeng Ying, Junji Wu. Research on on line monitoring system of overhead transmission line galloping,Science technology and Engineering, 2012, 12(14):3464-3466

[5] Kainan Li. Automatic interpretation of pointer type instrument based on Hough transform [J]. Modern electronic technology, 2006, 14: 18-19+26. 